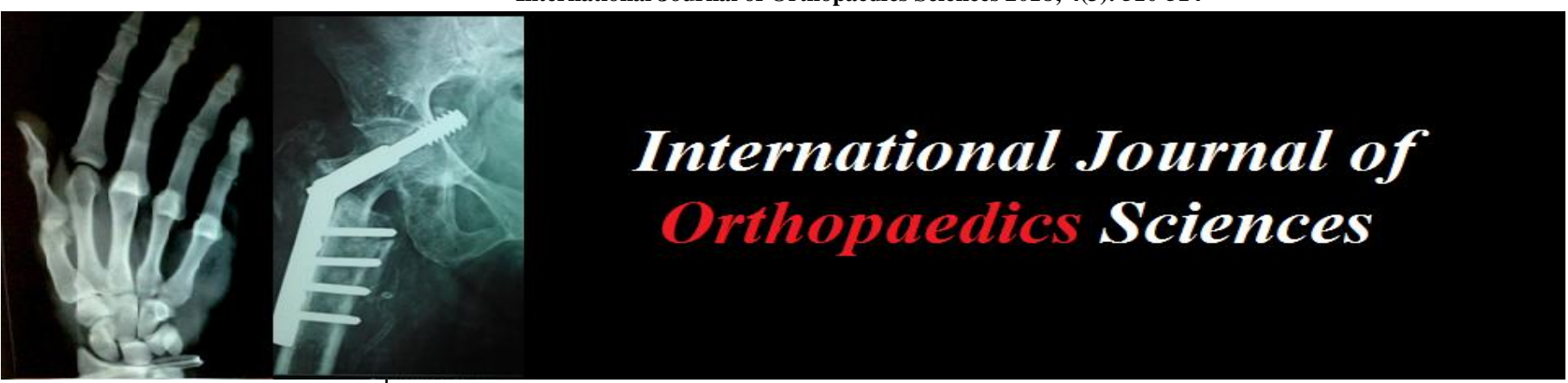

ISSN: $2395-1958$

IJOS 2018; 4(3): 510-514

(C) 2018 IJOS

www.orthopaper.com

Received: 24-05-2018

Accepted: 26-06-2018

Kumar Sandeep

Senior Resident, Swami

Dyanand Hospital, New Delhi,

India

Jagga Atul

Fellow Arthroplasty, Elite

institute of Orthopaedics, Max

Hospital Mohali. Punjab, India

Singh Hardev

Fellow Arthroscopy, Fortis

Hospital New Delhi.

Sharma Gaurav Kumar

GDMO-1, Swami Dyanand

Hospital, New Delhi, India

Dhiman Rajeev

Consultant, Apex Hospital and

Trauma Center Panipat,

Haryana, India

Adhikari Navneet

Senior Resident, PSRI Hospital,

New Delhi, India

\section{Kent Lalji}

Senior Consultant, Batra

Hospital and Medical Research

Centre, New Delhi, India
Correspondence

Kumar Sandeep

Senior Resident, Swami

Dyanand Hospital, New Delhi,

India

\section{Management of fracture shaft of humerus by dynamic compression plate versus interlocking nail: $\mathrm{A}$ prospective comparative study}

\author{
Kumar Sandeep, Jagga Atul, Singh Hardev, Sharma Gaurav Kumar, \\ Dhiman Rajeev, Adhikari Navneet and Kent Lalji
}

DOI: $\underline{\text { https://doi.org/10.22271/ortho.2018.v4.i3i.89 }}$

\section{Abstract}

Background and purpose: Simple humeral shaft fracture can be treated non operatively, with good results in most cases but the non-operative treatment requires a long period of immobilization, which carries a risk of prolonged shoulder joint stiffness and may be inconvenient for the patient. Furthermore, nonunion after conservative treatment of these fractures does occur in up to $10 \%$ of the cases and treatment of this condition can be very difficult. Now there is growing interest in treating even simple humeral shaft fractures by dynamic compression plate (DCP) or interlocking nail (ILN) in order to avoid these problems and to allow earlier mobilization and rapid return to work.

Methods: 40 patients of fracture shaft of Humerus were included in the study. 20 were managed by Interlocking Nail and 20 were managed by dynamic compression plate. Patients were called for follow up after every four weeks after the stitch removal and on each follow up visit, functional and radiological assessment done by predesigned performa. The functional results assessed at the end of 6 months and 1 year postoperatively on the bases of ASES Score (table 2), for 13 activities of daily living requiring full shoulder and elbow movement.

Results: At the end of study, 23 (92\%) cases in DCP group had acceptable (either good or more than good) results compared to $22(88 \%)$ cases of IMN group, having similar results. The mean interval between admissions to surgery, average operating time, duration of hospital study after surgery was less in DCP group while incidence of serious post-operative complications was more in IMN group. There was no statistically significant difference between the two groups with regards the average time taken for radiological union, although the mean radiological union in DCP group occurred 1.6 weeks earlier compared to the IMN group.

Interpretation: On the basis of this study, we found that DCP can be considered a better surgical option for the management of fracture of the shaft of humerus in our setting as it offers a shorter admission to surgery interval; shorter operating time and shorter hospital stay after surgery besides the lower incidence of more serious complication like the radial nerve palsy and infection otherwise comparable to IMN in all other variables of interest in our study.

Keywords: Management, fracture shaft, humerus, dynamic compression plate versus interlocking nail

\section{Introduction}

The humerus is divided into three parts as regards surgical anatomy; upper part, diaphysis and inferior part. The diaphysis or shaft can be defined as that part of the humerus situated between the superior margin of pectoralis major tendon insertion and $2 \mathrm{~cm}$ above olecranon fossa (Mast. et al. 1975, Varley 1995) [1]. Tytherleigh-Strong et al. (1998) [2] defined the supracondylar ridges as the lower border of the humeral shaft.

\section{Epidemiology of humeral shaft fractures}

A fracture of the shaft of humerus is a common occurrence. Approximately 5-10\% of all long bone fractures occur in the humerus (Rose et al. 1982) ${ }^{[3]}$. Humeral diaphyseal fracture account for about $20 \%$ of all humeral fractures (Rose et al. 1982) ${ }^{[3]}$. 


\section{Biomechanics}

Fractures of the humeral shaft can be caused by 3 broad mechanisms Direct, Indirect and through muscular forces.

Direct force implies an impact between the arm and an object creating a three point bending movement. This occurs when the patient falls against or is thrown against a fixed object or when a blunt object strikes the arm. This mechanism usually produces a transverse type fracture, occasionally with a non displaced butterfly fragment.

Indirect violence in which the energy absorbed by the humerus is applied through the distal portion of the limbs occurs in situations such as violent twisting of the arm behind the back or during arm wrestling. These rotational injures create a spiral fracture pattern.

Spiral fractures can also occur through muscular violence in activities such as throwing a baseball or a hand grenade.

Gunshot injuries represent yet another mechanism in which a high energy direct blow from a small projectile causes a highly comminuted fracture.

Certain predictable deformities result from muscle forces acting on fracture fragments. These deformities vary depending on the level at which fractures occurs. When fracture occurs above the insertion of pectoralis major, the proximal fragment is abducted and externally rotated by the rotator cuff muscles. Between the Pectoralis major and deltoid insertion, the proximal fragment displaces medially through the pull of Pectoralis major muscle. In fracture below the deltoid insertion, the strong deltoid muscle becomes the dominating force, abducting the proximal fragment and causing a varus deformity at the fracture site.

\section{Classification of humeral shaft fractures}

Humeral shaft fractures can be classified according to site (upper third, mid-third and lower third) or by fracture morphology (transverse, oblique, spiral, spiral wedge or segmental). The comprehensive AO classification is preferred in studies of humeral fractures. The reproducibility of comprehensive classification, however, may be questioned and usually only AO types (A, B or C) or main classes (e.g. A1-3) are reproducible enough to allow communication between orthopaedic surgeons.

\section{Complications}

Fractures of the shaft of humerus may be complicated with associated radial nerve palsy, delayed union, non-union or malunion.

Radial nerve palsy accompanies fracture of the humerus in $6 \%$ to $15 \%$ in various reports. It usually occurs in spiral fractures in the distal one third of the shaft (Holstein A and Lewis JB 1963) ${ }^{[8]}$ due to the anatomic proximity of the nerve to bone and also due to relative immobility of the radial nerve as it pierces the lateral intermuscular septum. Delayed or nonunion may occur after any method of treatment, closed or open. Factors such as insufficient immobilization, distraction of fracture site, and energy of the injury and open fractures have been some of the factors associated with non-union. Malunion and shortening are common following conservative modes of treatment but shortening up to $2 \mathrm{~cm}$ is not clinically evident and does not cause much of a functional disability.

\section{Treatment of humeral shaft fractures}

Although most uncomplicated humeral shaft fractures can be managed non-operatively, with an expected union rate of more than $90 \%$ using the methods which include the hanging cast, functional brace, Velpau dressing and shoulder spica cast, specific situations exists in which operative treatment is indicated like; open fractures, segmental fractures, pathological fractures, those with associated vascular injuries, bilateral humerus fractures, humerus fractures in polytrauma patients, radial nerve palsy after fracture manipulation, neurological loss after penetrating injuries, fractures with unacceptable alignment and failed conservative treatment.

Simple humeral shaft fracture can be treated non operatively, with good results in most cases (Watson-Jones 1955, Sarmiento et al. 2001) ${ }^{[9,10]}$, but the non-operative treatment requires a long period of immobilization, which carries a risk of prolonged shoulder joint stiffness and may be inconvenient for the patient (Rommens et al. 1995) ${ }^{[12]}$. Furthermore, nonunion after conservative treatment of these fractures does occur in up to $10 \%$ of the cases and treatment of this condition can be very difficult. Now there is growing interest in treating even simple humeral shaft fractures by dynamic compression plate (DCP) or interlocking nail (ILN) in order to avoid these problems and to allow earlier mobilization and rapid return to work (Rommens et al. 1995) ${ }^{[12]}$. The usual operative modalities used are the dynamic compression plate (DCP) and interlocking nail (ILN). Usually, fixation as achieved by a dynamic compression plate (DCP) is generally accepted that this gives satisfactory results. Use of this plate, however, requires extensive dissection and is complicated by the proximity of the radial nerve and the risk of mechanical failure in osteopenic bone. As a result of recent technical advances, there is growing interest in the use of the humeral interlocking nail (ILN) which can be inserted into the humerus antegrade, from the shoulder or retrograde, from the elbow. In theory, fixation by an ILN requires less invasive surgery and reaming can yield autograft material. The biomechanics are improved with higher moments of inertia and load-sharing capabilities but at the cost of postoperative shoulder pain and poor rotational control can lead to higher rates of non-union.

Selecting the right implant for internal fixation of humeral fractures remains controversial. Presently, to the best of the knowledge, there are only few published studies in the literature with limited number of patients which compare operative results between plating and interlocking nailing with contradictory conclusions.

\section{Material and Methods}

This study was conducted in the department of orthopaedics, Batra Hospital \& Medical Research Centre, New Delhi for a period of Aug 2013 to Jun 2015. 40 patients of fracture shaft of humerus were included in the study.

40 patients of fracture shaft of Humerus were included in the study.20 were managed by Interlocking Nail and 20 were managed by dynamic compression plate.

\section{Criteria for the inclusion into the study were:}

1. All displaced closed fractures $<3$ week old.

2. Type I and II open fractures reaching the hospital with in six hours of injury.

3. Patients in the age group of 18 to 70 years of both sexes.

\section{Exclusion criteria were the following:}

1. Type III open fracture

2. Type I and II open fractures reaching late

3. Pathological fractures

4. Complicated fractures with neurovascular injuries

5. Skeletally immature patients 
The Institution Review Board approved the study and the patients gave informed consent.

A complete history was taken and physical examination performed. Standard radiographs were obtained at presentation including anteroposterior (AP), lateral views. Clinical evaluation was done using a predesigned and pretested proforma with respect to history, clinical examination, radiological and functional assessment during preoperative and specific postoperative visits.

After complete pre-operative assessment and anesthetic clearance patients were randomized to receive either dynamic compression plating or interlocking nail for definitive fracture fixation.

The fractures were classified according to OA criteria (Table $1)$.

Table 1: Ao Classification of Humeral Shaft Fractures

\begin{tabular}{|c|c|}
\hline A - Simple Fracture & $\begin{array}{l}\text { A1 - Simple Fracture, Spiral } \\
\text { 1. Proximal Zone. } \\
\text { 2. Middle Zone. } \\
\text { 3. Distal Zone. A2. Simple Fracture, Oblique }\left(\mathbf{3 0}^{\mathbf{0}}\right) \\
\text { 1. Proximal zone } \\
\text { 2. Middle Zone } \\
\text { 3. Distal Zone A3 Simple Fracture, Transverse }\left(\mathbf{3 0}^{\mathbf{0}}\right) \\
\text { 1. Proximal Zone } \\
\text { 2. Middle Zone } \\
\text { 3. Distal Zone }\end{array}$ \\
\hline B. Wedge Fracture & $\begin{array}{l}\text { B1 Wedge Fracture, Spiral Wedge } \\
\text { 1. Proximal Zone } \\
\text { 2. Middle Zone } \\
\text { 3. Distal Zone } \\
\text { B2. Wedge Fracture, Bending Wedge } \\
\text { 1. Proximal Zone } \\
\text { 2. Middle Zone } \\
\text { 3. Distal Zone } \\
\text { 1. Proximal Zone } \\
\text { 2. Middle Zone } \\
\text { 3. Distal Zone }\end{array}$ \\
\hline C-Complex Fracture & $\begin{array}{l}\text { C1 Complex Fracture, Spiral } \\
\text { 1. With two intermediate fragments } \\
\text { 2. With three intermediate fragments } \\
\text { 3. With more than three intermediate fragments } \\
\text { C2 Complex Fracture, Segmental } \\
\text { 1. With one intermediate segmental fragment } \\
\text { 2. With one intermediate segmental and additional Wedge Fragment(s) } \\
\text { 3. two intermediate segmental fragments } \\
\text { C3 Complex Fracture, Irregular } \\
\text { 1. With two or three intermediate fragments } \\
\text { 2. With limited shattering }(<4 \mathrm{~cm}) \\
\text { 3. With extensive shattering }(>4 \mathrm{~cm})\end{array}$ \\
\hline
\end{tabular}

\section{Post-Operative Protocol}

The limb was kept in shoulder arm pouch just after operation and check X-rays were obtained for permanent record.

Range of motion exercises were started at shoulders and elbow joints as early as the third post-operative day, initially passive and later active as convenient.

Patients were discharged as soon as patient became comfortable, wound healthy, afebrile, satisfactory check Xray and were advised to return for removal of stitches at 2 weeks. During this period they were advised not to lift weight and do regular ROM exercises.

Patients were called for follow up after every four weeks after the stitch removal and on each follow up visit, functional and radiological assessment done by predesigned performa.

The functional results assessed at the end of 6 months and 1 year postoperatively on the bases of ASES Score (table 2), for 13 activities of daily living requiring full shoulder and elbow movement. 
Table 2: The American Shoulder and Elbow Surgeons (Ases) Score

\begin{tabular}{|c|}
\hline (ASES) score (4=normal: $\mathbf{3}=$ mild compromise: $\mathbf{2}=$ difficulty: $\mathbf{1}=$ with aid: $\mathbf{0 =}$ =unable). \\
Variables to measure \\
Back pocket Perineal care Wash opposite axilla \\
Eat with utensil Comb hair Use arm at shoulder level \\
Carry 10 Ib at side Dress Sleep on affected side \\
Pull Use hand over head Throw Lift \\
Excellent \\
The final results obtained using the ASES score, union time and ROM were graded as follow \\
ASES score $>40$ \\
Range of motion of shoulder or elbow within $10^{0}$ of the non-injured extremity in all planes. \\
Union in $<12$ weeks. \\
Good \\
ASES score $30-39$ \\
Painless shoulder abduction of $120^{\circ}$ \\
Union in $12-16$ weeks. \\
Poor \\
ASES score $<30$ \\
Union $>16$ weeks
\end{tabular}

\section{Statistical Methods Employed}

All data were statistically analyzed using MS Excel and SPSS 12.0 computer software for windows. The variables were represented as percentages (qualitative) and mean and standard deviation (quantitative). The statistical significance was assessed using $\mathrm{X}^{2}$ test/students test. A valve of $<0.05$ was considered as statistically significant. All $\mathrm{P}$ valves were two tailed. The analysis was carried out in accordance to intention to treat principle. evaluated for both groups and difference in $\mathrm{p}$ value is taken as parameter to decide which group is better than other.

\section{Results}

In our study, 20 cases of fracture shaft of humerus were treated with IMN and 20 more cases were treated with DCP.

The results observed were as follows:

- In both groups, the maximum number of patients were in the age group of 30-40 years.

- In both groups, there was male preponderance.

- In both groups, right extremity involvement was more common.

- Road Traffic Accidents were the main cause of fractures in both groups.

- Majority of the fractures in both the groups were AO / ASIF type A.

- In both groups, majority of the fractures were in the middle $3^{\text {rd }}$ of the shaft of humerus.

- Associated injuries including other long bone fractures etc. were common in both the groups.

- In our study, the admission to surgery interval was different for the two groups. In DCP group, the mean interval between admissions to surgery was 6.12 days whereas for IMN group, it was 11.88 days.

- In our study, the average operating time was different for the two groups. The average time taken for surgery was 50.8 minutes in cases of DCP group whereas in IMN group, it was 66.2 minutes.

- In our study the duration of hospital study after surgery was different for the two groups. The mean duration of hospital stay in DCP group was 8.76 days whereas in IMN group, it was 14.56 days.

- The two groups in our study differ with respect to the blood loss during surgery and other intra-operative difficulties/complications specific to the individual procedure itself.

- In our study, the incidence of serious post-operative complications was more in IMN group as compared to the DCP group.

- There was no statistically significant difference between the two groups with regards the average time taken for radiological union, although the mean radiological union in DCP group occurred 1.6 weeks earlier compared to the IMN group.

- The final results based on:

- ASES scores

- Union time and

- $\mathrm{ROM}$ of shoulder and elbow at the end of 24 weeks

- $23(92 \%)$ cases in DCP group had acceptable (either good or more than good) results compared to $22(88 \%)$ cases of IMN group, having similar results. There were 2 poor results in DCP group, one was a case of impingement and another was a case of delayed union. There were 3 cases in IMN group who had poor results; one of them was a case of radial nerve palsy and the other two cases had deep infection including one who later developed infected non union.

\section{Discussion}

On the basis of this study, we found that IMN can be considered a better surgical option for the management of fracture of the shaft of humerus in our setting as it offers a shorter admission to surgery interval; shorter operating time and shorter hospital stay after surgery besides the lower incidence of more serious complication like the radial nerve palsy and infection otherwise comparable to DCP in all other variables of interest in our study. The above and differences in our setting can be advantageous as it decreases the hospital workload and therefore the expenditure.

\section{References}

1. Mast JW, Spiegel PG, Harvey JP, Harrison C. Fractures of the humeral shaft. A retrospective study of 240 adult fractures. Clin Orthop. 1975; 112:254-262.

2. Tytherleigh-Strong G, Wallis N, McQueen MM. the epidemiology of humeral shaft fractures. J Bone Joint Surg. 1998; 80B:249-253.

3. Rose SH, Melton LJ, Morrey BF, IIstrup DM, Riggs BL. Epidemiological features of humeral fractures. Clin 
Orthop. 1982; 168:24-30.

4. Klenerman L. Fractures of the shaft of the humerus. J Bone Joint Surg. 1996; 48B:105-111.

5. Zimmerman MC, Waite AM, Deehan M, Tovey J, Oppenheim W. A biomechanical analysis of four humeral fracture fixation systems. J Orthop Trauma. 1994; 8:233239

6. McCormack RG, Brein D, Buckley RE, McKee MD, Rowell J, Schemitsch H. Fixation of fracture of the shaft of the humerus by dynamic compression plate or intramedullary nail. A prospective randomized trail. J Bone Joint Surg. 2000; 82B:336-339.

7. McKee MD, Miranda MA, Riemer BL, Blasier RB, Redmond BJ, Sims SH et al. Management of humeral nonunion after the failure of locking intramedullary nails. J Orthop Trauma. 1996b; 10:492-499.

8. Holstein A, Lewis GB. Fractures of the humerus with radial nerve paralysis. J Bone Joint Surg. 1963; 45A:1382-1388.

9. Watson-Jones R. Fractures and joint injuries. Livingston, Edinburgh, 1995.

10. Sarmiento A, Zagorski JB, Zych DO, Latta LL, Capps CA. Functional bracing for the treatment of fractures of the humeral diaphysis. J Bone Joint Surg. 2001; 82A:478-486.

11. Rodriguez-Merchan EC. Compression plating versus Hackenthal nailing in closed humeral shaft fractures failing nonoperative reduction. J Orthop Trauma. 1995; 9:194-197.

12. Rommens PM, Verbruggen J, Broos PL. Retrograde locked nailing of humeral shaft fractures. A review of 39 patients. J Bone Joint Surg. 1995; 77B:84-89. 